\title{
Editorial: Space Weather Prediction: Challenges and Prospects
}

\author{
Nandita Srivastava $^{1 *}$, Marilena Mierla ${ }^{2,3}$ and Jie Zhang ${ }^{4}$ \\ ${ }^{1}$ Udaipur Solar Observatory, Physical Research Laboratory, Udaipur, India, ${ }^{2}$ Solar-Terrestrial Centre of Excellence, Royal \\ Observatory of Belgium, Brussels, Belgium, ${ }^{3}$ Institute of Geodynamics of the Romanian Academy, Bucharest, Romania, \\ ${ }^{4}$ Department of Physics and Astronomy, George Mason University, Fairfax, VA, United States
}

Keywords: space weather, coronal mass ejections, stealth CMEs, geomagnetic storm, flux rope

\section{Editorial on the Research Topic}

\section{Space Weather Prediction: Challenges and Prospects}

Coronal mass ejections (CMEs), launched from the Sun are known to be the main drivers of space weather. Earth-directed CMEs can produce severe impacts upon their arrival at the Earth by compressing the magnetosphere and reconnecting with the Earth's magnetic field. Although space weather studies carried out in the last 2 decades have improved our understanding of solar drivers, i.e., CMEs and flares, however accurate prediction of the space weather impact of a given CME still eludes us.

Considerable progress has been achieved by researchers for estimation of arrival times with the development of semi-empirical/analytical models (Drag-Based Model); numerical simulations and Magnetohydrodynamic models (e.g., ENLIL, EUHFORIA), combined with observational techniques, such as interplanetary scintillation, wide-angle heliospheric imaging, and radio waves. However, there is no model developed yet that can accurately predict the arrival times and the geoeffectiveness of CMEs.

CMEs are considered to propagate as magnetic flux ropes from the Sun to the Earth so that the magnetic field of the CME can be predicted at any point in the inner heliosphere. However, due to the lack of the CME magnetic field observations close to the Sun, estimating the magnetic field orientation of the CME at $1 \mathrm{AU}$ is a major problem for forecasting. Furthermore, the impact of a single CME on the Earth's magnetosphere can be predicted to some extent with reliability. However, in the case of multiple CMEs, they may merge to form "complex ejecta," often resulting in severe space weather effects.

In this research topic, several important contributions related to prediction of space weather impact of CMEs i.e. arrival time and geoeffectiveness have been included. In addition, research contributions related to the early evolution of CMEs i.e., magnetic flux ropes; the initial width distributions of slow and fast CMEs which play an important role in impacting space weather conditions have been reported.

The research topic also includes an in-depth review by Vourlidas based on solar observations from a plethora of space missions, modelling efforts and on what has kept us holding back actionable medium term (hours to days) forecasting of space weather. The paper assesses 2 decades of research on solar drivers i.e., flux ropes observations in EUV and visible wavelengths from SOHO and SDO missions; multi-view point imaging of the Sun and tracking of the Earth-bound eruptions by twin STEREO spacecraft. The paper further highlights the bottle-necks in space weather forecasting and the current research gaps which include uncovering the magnetic configuration of the pre-eruptive structure; formation and dynamics of CMEs in the lower corona; propagation of CMEs in the interplanetary medium and its interaction with the ambient solar wind and CME-CME interaction. 
One of the main challenges for Space Weather prediction is understanding the coronal magnetic configuration of the preeruptive structure and how it leads to eruption which would help prediction of flares/CMEs. Inspite of several studies (Green et al., 2018), we are far from closing this challenge as the eruptions are magnetically driven and originate in the corona, where magnetic field measurement is difficult. A new approach is reported in this Research Topic by Kilpua et al. who implemented a fully datadriven and time-dependent magnetofrictional method (TMFM) developed by Pomoell et al. (2019), to model the early evolution of the flux rope using photospheric vector magnetograms as its sole boundary conditions. The method self-consistently produces a coherent flux rope and derives key magnetic properties such as twist, helicity, and axial magnetic flux. The axial magnetic flux value obtained from TMFM are in good agreement with observations, thereby promising a reliable space-weather forecast.

The Research Topic addresses a major challenge faced by the space weather forecasters due to Stealth CMEs which originate at higher altitudes in the corona, in regions with weaker magnetic fields and leave no signatures on the disk. To address this issue, have studied four stealth CMEs on which several image processing techniques were applied viz. image differencing, wavelets packets equalization, multi-scale Gaussian normalization and geometric techniques involving latitude projection, triangulation, GCS. The analysis shows that the main factor contributing to "stealthiness" is the spatial extent of the eruption rather than the altitude from which a CME lifts off. It is found that difference images with large temporal separations $(12 \mathrm{~h})$ revealed the clearest eruptive signatures. This technique proves to be promising for the early detection of stealth CMEs which can adversely impact the space weather.

An important parameter to predict is the time of arrival of CMEs which can be estimated from MHD modelling, numerical simulations and empirical tools. An example is the analytical drag based model (DBM) which is a simple and quick tool for heliospheric propagation of CMEs and CME arrival time prediction. In this research topic, Dumbović et al. provide an extensive overview of the assumptions, applications, and performance of the five sequentially developed DBM models for improved CME arrival time estimates. Out of these, three models basically differ on the assumed different initial geometries of CMEs. The other two approaches are based on ensemble models which provide an improved probability of arrival and confidence interval of the arrival time and speed. These lead to better predictions as they rely on a large number of computationally inexpensive DBM runs.

Understanding the propagation of CMEs before they arrive at the Earth is quite crucial for space weather prediction. This is achieved by studying the kinematical properties of CMEs using coronagraphic and heliospheric imaging observations, specifically by estimating the CME width. Research work reported here by Pant et al. shows that the width distribution of slow and fast CMEs follow different power law distributions that can be related to different types of source regions, viz. eruptive prominences and active regions; suggestive of different mechanisms involved in the width expansion of slow and fast CMEs originating from different sources.

Besides the arrival time estimates, one also needs to forecast if the Earth directed CMEs will be geo-effective. Besliu-Ionescu and Mierla report the results of an updated logistic regression model implemented on 2796 CMEs observed during solar cycle 24 for predicting their geoeffectivess. The model was trained $75 \%$ of these events and validated for the remaining $25 \%$. The model successfully predicted $98 \%$ of the events from the training set, and $98 \%$ of the events from the validation set. The study suggests that the model depends on CME properties derived close to the Sun and for robust analysis, one needs to incorporate the evolution of the CMEs in their heliospheric journey.

Solar Cycle 24 (SC24) was the weakest cycle in the last few decades and although solar wind reached its lowest values ever measured (McComas et al., 2013), the cosmic ray background reached record values. Weaker geomagnetic storms were recorded in SC24 (Manoharan et al., 2018) although the CME rate was largely the same (Lamy et al., 2017). The absence of strong events owes to the lower rate of fast and/or wide CMEs in SC24 (Gopalswamy et al., 2020). To understand the contribution of the heliospheric state on the expansion behavior of ICMEs, Mishra et al. estimated the dimensions of ICME structures at 1 AU during SC 23 and 24. They reported a decrease in the radial sizes of ICMEs at 1 AU in SC24 and also that the reduced heliospheric pressure in SC24 is compensated by the reduced magnetic content inside CMEs. Further, the average dimension of sheaths in both cycles is found to be equal, which is unexpected as the ICMEs in SC23 are stronger than that in cycle SC24. The study also highlights that one should consider the propagation and expansion speeds of the CMEs relative to the ambient solar wind speeds.

Lastly, future prospects in space weather prediction that are envisaged, have been emphasized by Vourlidas. These include the importance of coronal magnetic measurements; recording of uninterrupted observations from the solar surface to about 15 solar radii, and enhancing the capability of heliospheric imaging. Further, a more technological challenge to be addressed is planning an off -ecliptic heliospheric imaging. These efforts are expected to impact research and forecasting of space weather significantly.

\section{AUTHOR CONTRIBUTIONS}

All authors listed have made a substantial, direct, and intellectual contribution to the work and approved it for publication. 


\section{REFERENCES}

Gopalswamy, N., Akiyama, S., and Yashiro, S. (2020). The State of the Heliosphere Revealed by Limb-Halo Coronal Mass Ejections in Solar Cycles 23 and 24. Astrophysical J. Lett. 897, L1. doi:10.3847/2041-8213/ab9b7b

Green, L. M., Török, T., Vršnak, B., Manchester, W., and Veronig, A. (2018). The Origin, Early Evolution and Predictability of Solar Eruptions. Space Sci. Rev. 214, 46. doi:10.1007/s11214-017-0462-5

Lamy, P., Floyd, O., Quémerais, E., Boclet, B., and Ferron, S. (2017). Coronal Mass Ejections and Solar Wind Mass Fluxes Over the Heliosphere During Solar Cycles 23 and 24 (1996-2014). J. Geophys. Res. Space Phys. 122, 50-62. doi:10.1002/2016JA022970

Manoharan, P. K., Mahalakshmi, K., Johri, A., Jackson, B. V., Ravikumar, D., Kalyanasundaram, K., et al. (2018). Current State of Reduced Solar Activity: Intense Space Weather Events in the Inner Heliosphere. Sun and Geosphere. 13, 135-143. doi:10.31401/SunGeo.2018.02.03

McComas, D. J., Angold, N., Elliott, H. A., Livadiotis, G., Schwadron, N. A., Skoug, R. M., et al. (2013). Weakest Solar Wind of the Space Age and the Current "Mini" Solar Maximum. Astrophys. J. 779, 2. doi:10.1088/0004$637 \mathrm{X} / 779 / 1 / 2$
Pomoell, J., Lumme, E., and Kilpua, E. (2019). Time-Dependent Data-Driven Modeling of Active Region Evolution Using Energy-Optimized Photospheric Electric fields. Sol. Phys. 294, 41. doi:10.1007/s11207-019-1430-x

Conflict of Interest: The authors declare that the research was conducted in the absence of any commercial or financial relationships that could be construed as a potential conflict of interest.

Publisher's Note: All claims expressed in this article are solely those of the authors and do not necessarily represent those of their affiliated organizations, or those of the publisher, the editors and the reviewers. Any product that may be evaluated in this article, or claim that may be made by its manufacturer, is not guaranteed or endorsed by the publisher.

Copyright (c) 2021 Srivastava, Mierla and Zhang. This is an open-access article distributed under the terms of the Creative Commons Attribution License (CC BY). The use, distribution or reproduction in other forums is permitted, provided the original author(s) and the copyright owner(s) are credited and that the original publication in this journal is cited, in accordance with accepted academic practice. No use, distribution or reproduction is permitted which does not comply with these terms. 